\title{
Responding to the maternal health care challenge: The Ethiopian Health Extension Program
}

\author{
Marge Koblinsky, Frances Tain, Asheber Gaym, Ali Karim, Mary Carnell, Solomon Tesfaye
}

\begin{abstract}
Background: Responding to challenges in achieving Millennium Development Goals (MDG), the Ethiopian government initiated the Health Extension Program in 2003 as part of the Health Sector Development Program (HSDP) to improve equitable access to preventive, promotive and select curative health interventions through paid community level health extension workers.

Objective: To explore Ethiopia's progress toward achieving MDG 5 that focuses on improved maternal health through the Health Extension Program.

Methods: This paper reviews available survey data and literature to determine the feasibility of reaching the targets specified for MDG 5 and for HSDP.

Important findings: Achieving the set targets is a daunting task despite reaching the physical targets of two health extension workers per kebele. The 2015 MDG target for the Maternal Mortality Ratio (MMR) is 218 while the 2005 MMR estimate is 673 . The HSDP target is $32 \%$ skilled birth attendant use by 2010 but only about $12 \%$ use was found in the four most populated regions of the country in 2009.

Conclusions: Accelerating progress towards these targets is possible through the Health Extension Program at the worker level through improved promotion of family planning and specific maternal interventions, such as misoprostol for active management of third stage of labor, immediate postpartum visits, and improved coordination from community to referral level. [Ethiop. J. Health Dev. 2010;24 Special Issue 1:105-109]
\end{abstract}

\section{Introduction}

With a maternal mortality ratio of 673 (1) and 19,000 maternal deaths annually, Ethiopia is a major contributor to the world-wide death toll of mothers. Direct causes of mortality are similar to other developing countries (2) unsafe abortion, obstructed labor, puerperal sepsis, eclampsia and hemorrhage. The potential for a substantial level of maternal deaths from indirect causes is high as malaria, HIV/AIDS, tuberculosis, and poor nutrition are prevalent but no data are available. Low use of all maternal health services exacerbates the potential health consequences in 2005, use of skilled birth attendance was $6 \%$; cesarean section $1 \%$; postnatal care $6 \%$ and contraceptive prevalence rate $14 \%$ (1).

Behind these service statistics are barriers at all levels. Essential services for rural mothers are rare with facilities and skilled providers few and far $(3,4)$. Cultural beliefs, costs of care, lack of transportation/distance, lack of decision making power by women and desire for community ownership, contribute to lack of use $(5,6)$. Nearly $90 \%$ of delivering women are cared for by relatives and neighbors $(55 \%)$ and traditional birth attendants when available (33\%) (1).

Yet the Ethiopian Government is a strong advocate for improved maternal health as evinced by its commitment to the Millennium Development Goals (MDGs). It is also committed to improvement of maternal health as a primary goal of the Health Sector Development Program (HSDP III). The key vehicles to achieve these goals are the Health Extension Program (HEP), introduced in 2003, plus referral support. This paper outlines the plan for the maternal health component of HEP and progress towards national goals in the most modest scenario of HSDP III-- reduction of the MMR from 871 to 600 and increased skilled birth attendance from $9 \%$ to $32 \%$ by 2010 (7).

\section{Background}

Ethiopia, one of the most populous countries in subSaharan Africa (74.5 million, 2007) (8) yet one of the least urbanized (16\%) (8), and poorest (per capita gross national income of US \$220) (9), has focused on community-based preventive and promotive interventions since 1993. Within the Health Sector Development

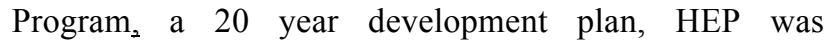
introduced in 2003 to respond to the acknowledged challenge that few people had access to modern health care and communicable diseases were extremely prevalent. Patterned after the Agricultural Extension Program, HEP is to provide equitable access to promotive, preventive and select curative health interventions through 30,000 government-salaried health extension workers (HEWs), two per kebele, located at a health post.

The HEWs, young local women with grade 10 education, are recruited by Kebele and Woreda Councils and given one year training prior to employment with the Woreda Health Office. Supporting HEP is the government's fourtiered health system with primary units (health center with 5 satellite health posts), and woreda hospitals, zonal hospitals, and specialized referral hospitals for populations of 25,000, 250,000, 1,000,000 and 5,000,000 respectively (10).

HEWs are to spend $75 \%$ time for outreach, primarily health promotion, with services at both health post and outreach sites. Among the services based on 16 health packages, maternal health is part of the Family Health package and includes delivery of normal births. About

John Snow, Inc., 1616 N Fort Myer Drive, Arlington, Virginia, 22209, Tel: 1-703-528-7474, Fax: 1-703-528-7480,

Email: mkoblinsky@jsi.com 
twenty voluntary community health workers may support HEW activities per kebele. At least two diploma level midwives and one health officer with emergency obstetric care training are to support HEWs from the local health center.

Decentralization is key to HEP's implementation: Woredas are to receive a block grant to cover expenditures to effect and manage services, and Kebele Councils plan and implement the programme.

\section{Methods}

Using mesh and key words (maternal mortality or maternal health services or maternal-child health centers or pregnancy complications or "safe motherhood" or maternal morbidity or maternal morbidities or postpartum complications and Ethiopia), we retrieved 276 abstracts from PubMed, of which 26 articles were used. Documents on the Ethiopian Federal Ministry of Health (FMOH) website and others from the FMOH were also reviewed.

The 2008/09 L10K project survey results are based on interviews in Oromia, SNNP, Tigray, and Amhara of 2,448 mothers of children 0-11 months old asking about their use of services; family planning data were collected on a sample of 4,080 women of reproductive age (11). These results are compared with maternal health care use indicators in project areas in 2005 obtained from secondary analysis of the Ethiopian Demographic and Health Surveys (EDHS) 2005 data (11).

The ESHE project household surveys were regional representative surveys conducted in Amhara, Oromia and SNNP in April-June, 2008 (12) and in 2003 (13); these two survey periods used the same sampling design and questionnaire, asking mothers about their pregnancy and birth of a child born in the previous year $(12,13)$.

\section{Results}

\section{The status of HEP through mid-2009}

Five years into implementation, few studies have published findings on HEP. In 2004, training of the first batch of HEWs was found wanting in terms of training sites (inadequate classrooms, libraries and basic infrastructure), training materials (in English and hence not well understood, no practical training, no demonstration models) and HEW selection (not from rural areas, low educational scores) (14).

A 2006 study of 60 HEWs from the first training batch posted at least 6 months in six areas of the country, found $25 \%$ health posts had no protected water and $16 \%$ no toilet (15). Most HEWs were isolated from health facilities, with little access to taxis, telephone or radio. Contrary to plan, over $50 \%$ of HEWs came from urban areas; only $8 \%$ were from the kebele they were serving. Their work focused on health education and some on environmental health. Family health services, including deliveries, were not implemented; $40 \%$ had made referrals but received no feedback. However, supervision was carried out typically on a three monthly basis as planned (15).

Relations with the kebele administration and with community workers were unclear. Few HEWs had carried out community surveys originally planned as the basis for work. Curative care was the most pervasive need felt by the community, but not a major function of HEWs (15).

In remote Tigray a year into implementation $58 \%$ of 60 female heads-of-household randomly selected, said their HEW was helpful and 93\% preferred an HEW over a Traditional Birth Attendant (TBA) to assist them during delivery because they seemed more knowledgeable and accessible (16). Only $85 \%$ received monthly or less frequent visits by HEWs, and only $13 \%$ reported discussion of contraception (16).

In 2008, mid-term reviewers of HSDP III noted the major HSDP objective of universal primary health care coverage by $2007 / 8$ was achievable: 24,500 of the 2010 target of 30,000 HEWs were trained (17). Regarding maternal health reviewers stated: "In maternal health, there is need to limit the scope of functions that is expected of the HEWs." (pg xix). They stated that HEWs should support TBAs with utilities and training to improve delivery skills and care but HEWs should not be expected to provide delivery care. They noted that the HSDP policy of fee exemption for pregnant women and young children for both services and supplies had not been implemented, making service use by the poor hard. Especially problematic were hospital costs, specifically for cesarean section (17).

Referral support from midwives at health centers for the HEWs was mentioned - only 685 of the required 3200 health centers were constructed by 2008 and staffing inadequacies -- only 1290 functioning midwives or 1 per 2171 births nationally -- were obvious. A more recent survey found less than half available hospitals and health centers provided basic and emergency obstetric care (BEmOC and EmOC) and only 174 obstetricians/gynecologists and fewer anesthetists were available nationwide (18).

By May 2009 the FMOH reported 30,193 HEWs deployed and nearly 11,000 of the required 15,000 health posts constructed (73\%) (19). An independent sample survey (2008/9) in Tigray, Amhara, Oromia, and SNNP found that by the end of $2008,76 \%$ of kebeles had the planned two HEWs in place and $69 \%$ the health post (11). Over $50 \%$ of health posts had a delivery couch and delivery kits but maternal health care supplies (e.g., iron tablets and ergometrine) were less available (11). Nearly $20 \%$ HEWs had received a 4 week in-service training including safe clean delivery and a quarter had training in

Ethiop. J. Health Dev. 2010;24 Special Issue 1 
essential neonatal health care (ENHC). HEWs reported that little work time was used on these services a median 2 hours per week for delivery services and 1 hour on ENHC (11). Environmental education and immunization required the majority of their time.

\section{Use of maternal health care and family planning}

In 2009 in L10K areas $12 \%$ women reported using any health professional for delivery and $4 \%$ a HEW. These levels compare favorably with ESHE 2008 statistics of $7 \%$ and $2 \%$ respectively in three of four $\mathrm{L} 10 \mathrm{~K}$ regions, and $5 \%$ professional care use in $2005(1,11)$. Institutional deliveries follow the pattern of professional provider use: in L10K areas institutional deliveries were reported by $9 \%$ of mothers with a child under one year, by $7 \%$ in the ESHE 2008 survey and by $5 \%$ in 2005 (Table 1).
Any antenatal care (ANC) use has improved substantially: The rate for any ANC visit is about 54\% in L10K areas, similar to the ESHE 2008 survey, and up from $26 \%$ in $\mathrm{L} 10 \mathrm{~K}$ areas in 2005 . Tetanus protection also increased to about $42-45 \%$ from $24 \%$ in 2005 . The WHO recommended four ANC visits remained relatively lowcurrently about $20 \%$ compared with $12 \%$ in 2005 in the same areas.

Postnatal care remained low at 7\% in $\mathrm{L} 10 \mathrm{~K}$ areas and unchanged from 2005 in the same areas; most of this care occurred after the first week of life when it is most needed to save lives of mothers and newborns (Table 1).

Improvements in the contraceptive prevalence rate (CPR) exceeded rates of maternal care use with the exception of antenatal care: Amongst married women of reproductive age, there was a $32 \%$ rate in $2008 / 9,29 \%$ reported in 2008 and a 16\% CPR in L10K areas in 2005 (Table 1).

Table 1: Ethiopia National Maternal Health Trends (\%), 2005, 2008/9

\begin{tabular}{llll} 
& $\begin{array}{l}\text { L10K areas, } \\
\text { DHS 2005 } \\
(1,11)\end{array}$ & $\begin{array}{l}\text { USAID/ESHE 2008 (12) } \\
\text { 3 Regions } \\
\text { weighted avg. }\end{array}$ & $\begin{array}{l}\text { L10K 2008/09 (11) } \\
\text { 4 Regions } \\
\text { weighted avg. }\end{array}$ \\
\hline FP (married women 15-49 years) & & & \\
Any method & 16 & 29 & 32 \\
Modern method & 15 & 25 & 32 \\
ABC & & & \\
Any & 26 & 56 & 54 \\
4+ visits & 12 & - & 20 \\
TT2+ & 24 & 45 & 42 \\
Fe/folate & 10 & 28 & 15 \\
Delivery & & & 12 \\
Health Professional & 5 & 7 & 4 \\
HEW & - & 2 & 8 \\
Trained TBA & - & 11 & 12 \\
TBA & 20 & 41 & 9 \\
At health facility & 5 & 7 & 91 \\
At home & 95 & 93 & - \\
C-section & 1 & - & 7 \\
Postpartum Care/Post Natal Care & & & 2 \\
Any & 7 & 8 & 3 \\
<2 days & 5 & - & 5 \\
By health professional & 6 & 7 & \\
By HEW & - & - & \\
\hline = data not available & & & \\
& & &
\end{tabular}

\section{Discussion and Conclusions}

Ethiopia is rich in publicly stated commitments and policies aimed at reduction of maternal mortality. But achieving the goals of HSDP III or targets for MDG 5 by 2015 is unlikely. The MMR goal for 2010 of 600 is close to the 2005 reported figure of $673 / 100,000$ live births but far from the 218 MDG 5 target for 2015.

Increasing skilled birth attendance to $32 \%$ by 2010 from the 2009 level $(12 \%)$ is not likely even though a health post staffed by HEWs in every rural village will shortly be attained. While these data have limitations, they are representative for the areas of interviews.
Expectations that HEWs fill the void of skilled birthing care are optimistic. HEWs have minimal training and virtually no hands-on training in skills needed for normal birthing, let alone the first aid needed to stabilize a woman for referral. Secondly they have a variety of other tasks that require their time and for which they are likely better prepared, including household and community visits for health promotion efforts.

EmOC care is known to directly contribute to maternal mortality reduction (20). Yet functioning health centers and hospitals and the skilled providers to staff them midwives, specialists, and trained health officers - are not yet available at levels needed.

Ethiop. J. Health Dev. 2010;24 Special Issue 1 
However HEWs can effectively provide the following community level services to reduce maternal deaths now:

- Family planning with a broad method mix and follow up care to ensure continuity. With an approximate $34 \%$ unmet need for family planning (1), there is obvious room for improvement in contraceptive use.

- Hygienic delivery through partnership with TBAs with the TBA catching the baby and the HEW providing needed newborn care.

- Active Management of Third Stage of Labor with an active uterotonic post-delivery (e.g., misoprostol).

- An immediate post-partum visit to the mother and newborn to ensure breastfeeding support and referral if needed.

- Continued promotion of birth preparedness, complication readiness.

- Knowledge of and communication means with a referral center.

Increasing skilled attendance at birth requires skills in normal delivery and first aid if complications arise. Nurses, more available than other health care providers, could be provided on the job training in midwifery skills, specifically stabilization of women with bleeding and assisted delivery for prolonged labor.

Referral support is slowly being established. Maternity waiting homes could provide women with little availability to transport, to be in the vicinity of a referral center at delivery time. Such may be most useful for women with a prior perinatal death or first birth. Yet a maternity waiting home alone is not the answer and obviously must be attached to a functioning referral center.

Specific gaps, if filled, could ensure major direct causes of maternal mortality were addressed relatively quickly:

- Unsafe abortion deaths could be reduced with expansion of use of Manual Vacuum Aspirators (MVA) in the hands of trained personnel. HEWs' training should include Comprehensive Abortion Care.

- Use of iron folate tablets to decrease maternal anemia is recommended in international guidelines, and supplies to HEWs are much needed.

Building demand remains a major challenge, but there are opportunities that could be availed:

- Household decision making remains with the husband, especially where money is concerned. Ensuring men are involved in birth planning, complication readiness efforts is much needed.

- Costs of delivery, specifically cesarean sections, remain high. Reducing costs or providing vouchers or social insurance for the poor, may make services more attractive.
- Prolonged/obstructed labor is a major cause of maternal and newborn death and disability (including fistulas). Developing awareness and recognition of this particular problem when it occurs is much needed along with provider skills to provide assisted delivery (or referral for surgery) for such women.

Ethiopia has set the groundwork to improve maternal health with its strong commitment to maternal health in policies, and HEP's success in reaching communities. Accelerating this pace, drawing upon recommendations of the HSDP III Mid-term Review and Ethiopian experts at the 2009 Workshop on Maternal and Newborn Health, is much needed to move toward the set goals.

\section{Acknowledgement}

We appreciated The Bill and Melinda Gates Foundation (grant number 49114) support for this work.

\section{References}

1. Central Statistical Agency (Ethiopia) and ORC Macro. Ethiopia Demographic and Health Survey 2005. Addis Ababa, Ethiopia and Calverton, Maryland, USA: Central Statistical Agency and ORC Macro, 2006.

2. Ronsmans C, Graham WJ. Maternal mortality: who, when, where, and why. Lancet 2006;368(9542):1189-200.

3. Girma S, Yohannes A, Kitaw Y, et al. Human Resource Development for Health in Ethiopia: Challenges of Achieving the Millennium development Goals. Ethiop .J Health Dev 2007;21(3):216-231.

4. Gaym A, Worku B, Getaneh E, Berhe A, Tiruneh T. Report on the National Situational Analysis of PreService Midwifery Training in Ethiopia. Addis Ababa: Addis Ababa University and WHO, 2008.

5. Ethiopia Federal Ministry of Health. Report on the Safe Motherhood Community-Based Survey, Ethiopia. Addis Ababa: Family Health Department, 2006.

6. Koblinsky M, Tain F, Tesfaye S, Betamariam W. Working paper 1: Reducing maternal mortality and increasing use of skilled birth attendance: Ethiopia and MDG 5 - status as of 2005, 2009.

7. Ethiopia Federal Ministry of Health. Health Sector Strategic Plan (HSDP-III), 2005/6-2009/10. Addis Ababa: Planning and Programming Department, 2005.

8. Federal Democratic Republic of Ethiopia Population Census Commission. Summary and Statistical Report of the 2007 Population and Housing Census Results. Addis Ababa, 2008.

9. World Bank. World Development Indicators database; gross national income per capita 2007, atlas method. p. 2007. 
10. Ethiopia Federal Ministry of Health, Planning and Programming Department. Health and Health Related Indicators, 1999 E.C (2006/07 G.C.). Addis Ababa: FMOH, 2007.

11. The Last Ten Kilometers Project. Baseline Household Health Survey: Amhara, Oromiya, SNNP, and Tigray. JSI Research \& Training, Inc., Addis Ababa, Ethiopia. 2009.

12. USAID/Essential Services for Health in Ethiopia (ESHE). Household End-line Survey Synthesis Report. Addis Ababa, 2008.

13. USAID/Essential Services for Health in Ethiopia (ESHE). Twelve Baseline Health Surveys, Conducted in: Amhara, Oromia and Southern Nations, Nationalities \& Peoples' Regions. Addis Ababa, 2005.

14. Kitaw Y, Ye-Ebiyo Y, Said A, Desta H, Teklehaimanot A. Assessment of the Training of the First Intake of Health Extension Workers. Ethiop J Health Dev 2007;21(3):232-239.

15. Eklehaimanot A, Kitaw Y, G/Yohannes A, et al. Study of the Working Conditions of Health Extension Workers in Ethiopia. Ethiop J Health Dev 2007;21(3):246-259.
16. Negusse H, McAuliffe E, Maclachlan M. Initial community perspectives on the Health Service Extension Program in Welkait, Ethiopia. Hum Resour Health 2007;5:21.

17. HSDP III Independent Review Team. Ethiopia Health Sector Development Program (HSDP III) Mid-Term Review, Volume I Component Report. Addis Ababa, 2008.

18. Kwast B. Preliminary results from the national EmONC baseline assessment 2007- 2008 by FMOH, UNICEF, UNFPA, WHO, AMDD. National Workshop to Address Community Maternal and Neonatal Health in Ethiopia. Addis Ababa, 2009.

19. Messelech A. All Roads Lead to Health Extension Program. National Workshop on Addressing Community Maternal \& Neonatal Health in Ethiopia. Addis Ababa, 2009.

20. Chowdhury M, Ahmed A, Kalim N, Koblinsky M. Causes of maternal mortality decline in Matlab, Bangladesh. J Health Popul Nutr 2009;27(2):108123. 